\title{
Bile acids induce apoptosis selectively in androgen-dependent and -independent prostate cancer cells.
}

Prostate cancer is a prevalent age-related disease in North America, accounting for about $15 \%$ of all diagnosed cancers. We have previously identified lithocholic acid (LCA) as a potential chemotherapeutic compound that selectively kills neuroblastoma cells while sparing normal human neurons. Now, we report that LCA inhibits the proliferation of androgen-dependent (AD) LNCaP prostate cancer cells and that LCA is the most potent bile acid with respect to inducing apoptosis in LNCaP as well as androgen-independent (AI) PC-3 cells, but without killing RWPE-1 immortalized normal prostate epithelial cells. In LNCaP and PC-3 cells, LCA triggered the extrinsic pathway of apoptosis and cell death induced by LCA was partially dependent on the activation of caspase-8 and -3. Moreover, LCA increased cleavage of Bid and Bax, down-regulation of Bcl-2, permeabilization of the mitochondrial outer membrane and activation of caspase-9. The cytotoxic actions of LCA occurred despite the inability of this bile acid to enter the prostate cancer cells with about $98 \%$ of the nominal test concentrations present in the extracellular culture medium. With our findings, we provide evidence to support a mechanism of action underlying the broad anticancer activity of LCA in various human tissues. 
6 Alexander A. Goldberg ${ }^{1}$, Vladimir I. Titorenko ${ }^{2}$, Adam Beach ${ }^{2}$, J. Thomas Sanderson ${ }^{1 *}$

$8 \quad{ }^{1}$ INRS-Institut Armand-Frappier, Laval, Québec, Canada.

$9 \quad{ }^{2}$ Department of Biology, Concordia University, Montréal, Canada.

11 " $P l e a s e$ address correspondence to: Thomas Sanderson, 531 boulevard des Prairies, Laval, H7V 12 1B7, QC, Canada. 


\section{Introduction}

Prostate cancer accounts for approximately 15\% of all newly diagnosed cancers and it is the third highest cause of cancer-related deaths in males in the United States . Most prostate cancers are initially androgen-dependent (AD) and are generally treated with a combination of radiotherapy, chemical castration, androgen-receptor (AR) antagonists (hydroxyflutamide, bicalutamide), inhibitors of steroidogenesis (abiraterone). However, a large contingent of AD cancers will progress to become a more aggressive, androgen-independent (AI) form, and less readily treatable, resulting in a higher incidences of morbidity and mortality. Furthermore, patients treated with either hydroxyflutamide or bicalutamide are known to suffer from severe side-effects as a result of the anti-androgenic therapy, necessitating the search for natural and more potent anti-cancer compounds with fewer deleterious effects on the human body.

Bile acids are the products of cholesterol catabolism and their main function for the body is the solubilisation of dietary fats and fat-soluble vitamins from the intestinal lumen . More recently, bile acids have been found to have specific regulatory functions, as they interact with a variety of intracellular and extracellular signalling molecules such as the farnesoid X (FXR), vitamin D (VDR), pregnane X (PXR) and G-protein coupled (TGR5) receptors. Numerous studies have shown that bile acids play key homeostatic roles in glucose metabolism, cholesterol and lipid metabolism, xenobiotic detoxification of toxins and lifespan extension . Moreover, BAs can be useful small molecules for the treatment of illnesses such as cholestatic liver disease, Alzheimer's disease, atherosclerosis, obesity and metabolic disorders . It has also been reported that bile acids have anti-neoplastic and -carcinogenic properties in a multitude of cancer cell models, such as tamoxifen-resistant breast cancer, colon cancer , prostate cancer and neuroblastoma cells.

We have previously reported that LCA delays chronological aging of the budding yeast, $S$. cerevisiae, independent of AMP-activated protein kinase/target of rapamycin (AMPK/TOR) and cAMP/Protein Kinase A (PKA) signalling. LCA alters the age-related dynamics of metabolomic processes in yeast such as respiration and reactive oxygen species production in the mitochondria, and lipid and trehalose accumulation, and modulates stress response pathways . Moreover, we have shown that LCA can kill human neuroblastoma cells, while sparing normal human primary neurons. LCA selectively initiates an extrinsic apoptotic programme of cell death 
43 in neuroblastoma cells, thus recruiting and activating the initiator caspase-8, inducing

44 mitochondrial outer membrane permeabilization (MOMP), mitochondrial fragmentation, and

45 ultimately activation of the downstream proteases caspase- 9 and -3 . Here we report that bile

46 acids can inhibit dihydrotestosterone (DHT)-induced cell proliferation, and kill both AD and AI

47 prostate cancer cells in a caspase-3 dependent manner, by eliciting the intrinsic and extrinsic

48 pathways of apoptosis. The mechanistic studies we present will further our understanding of the

49 potential of bile acids to act as chemotherapeutic agents against prostate cancer.

50

51 Materials and Methods:

52 Cell lines and reagents.

LNCaP, PC-3 and RWPE-1 cell lines were purchased from ATCC (Manassas, VA). LNCaP 54 and RWPE-1 cells were grown in RPMI 1640 supplemented with 10\% fetal bovine serum or 2\% 55 dextran-coated charcoal-stripped FBS, 2 mM L-glutamine, 1\% HEPES, 1\% sodium-pyruvate and $10 \mathrm{ml} / \mathrm{L}$ of 100x antibiotic-antimycotic solution. PC-3 cells were grown in a 1:1 mixture of DMEM and Ham’s F12 Nutrient Mixture with either 10\% fetal bovine serum or 2\% dextran-coated charcoal-stripped FBS, 2 mM L-glutamine and $10 \mathrm{ml} / \mathrm{L}$ of 100x antibiotic-antimycotic solution (Sigma-Aldrich, St-Louis, MO). Cells were maintained in a humidified atmosphere $\left(5 \% \mathrm{CO}_{2}\right)$ at $37^{\circ} \mathrm{C}$. Lithocholic acid (LCA), deoxycholic acid (DCA), chenodeoxycholic acid (CDCA), ursodeoxycholic acid (UDCA), hyodeoxycholic acid (HDCA) and cholic acid (CA) were purchased from Sigma-Aldrich and dissolved in DMSO to make 500 mM stock solutions. Dihydrotestosterone (DHT; Steraloids Inc., Newport, RI) was dissolved in DMSO to make $100 \mathrm{mM}$ stock solutions. The final concentration of DMSO in culture medium was not greater than $0.2 \%$. The selective caspase substrates Ac-DEVD-AFC, Ac-IETD-AFC and Ac-LEHD-AFC were purchased from Enzo Life Sciences (Farmingdale, NY) and dissolved in DMSO to make $20 \mathrm{mM}$ stock solutions. Caspase inhibitors z-DEVD-fmk and z-IETD-fmk (BD Biosciences, Franklin Lakes, NJ) were dissolved in 100\% DMSO to produce $10 \mathrm{mM}$ stock solutions. All primary antibodies were purchased from Cell Signaling (Beverly, MA). LNCaP and PC-3 cells were exposed to 1000-fold dilutions of the appropriate stock solutions of bile acids, DHT and/or caspase inhibitors in their respective experimental culture media. Control cells were exposed to $0.1 \%$ or $0.2 \%$ DMSO for single or co-exposure experiments, respectively. 


\section{LNCaP cell proliferation.}

LNCaP cells were seeded in 16-well E-plates (Roche Diagnostics, Laval, QC) at a density of $25 \times 10^{3}$ cells per $200 \mu \mathrm{l}$ medium containing 2\% stripped FBS/well. After 24 hours, DHT was added at a concentration $(0.1 \mathrm{nM})$ that stimulated optimal growth rate (avoiding surpassing confluence) in culture over a $72 \mathrm{~h}$ period along with various concentrations of LCA or DMSO vehicle. Then cell proliferation was determined quantitatively and in real-time over a period of 72h by measuring changes in impedance detected by the gold electrode-microarrays at the bottom of each of the 16 wells of the E-plate.

Apoptosis, necrosis and mitochondrial membrane potential.

For apoptotic and necrotic cell death measurements , LNCaP and PC-3 cells were seeded at densities of $1 \times 10^{5}$ and $0.5 \times 10^{5}$ cells/ml, respectively, in 24-well plates in $2 \%$ stripped-FBS. Cells were then treated with several concentrations of bile acids in the presence (LNCaP) or absence (PC-3) of 0.1 nM DHT. After 48 hours, Hoechst 33342 (Sigma-Aldrich) and propidium iodide

(PI; Invitrogen, Carlsbad, CA) were each added at a concentration of $1 \mu \mathrm{g} / \mathrm{ml}$ per well. After a 15 minute incubation at $37^{\circ} \mathrm{C}$, cells were observed and counted under a Nikon Eclipse (TE-2000U) inverted fluorescent microscope at 20x magnification. Hoechst-positive and PI-positive cells were made visible using filter cubes with excitation wavelengths of 330-380 nm and 532-587 $\mathrm{nm}$, respectively. To measure mitochondrial membrane potential (MMP), cells were treated with LCA for 1, 4 or 8 hours, and tetramethylrhodamine ethyl ester (TMRE) was added to each well at a final concentration of $50 \mathrm{nM}$. TMRE is a cell permeable, positively charged dye that accumulates in active negatively charged mitochondria . In inactive or depolarized mitochondria, membranes have decreased potential and fail to sequester TMRE. After a 15 minute incubation at $37^{\circ} \mathrm{C}$, cells were observed under an inverted fluorescent microscope using a filter cube with excitation wavelengths of 532-587 $\mathrm{nm}$. The photos were then analyzed using ImageJ image processing software .

Caspase activity assays.

PC-3 and LNCaP cells were seeded in 6-well plates at densities of 400000 or 750000 cells per well, respectively, in $0.5 \mathrm{ml}$ culture medium containing $2 \%$ stripped FBS and 24 hours later they were exposed to various concentrations of LCA in fresh medium for another 24 hours. Proteins were then extracted from harvested cells using 1x RIPA buffer (Millipore, Billerica, 
$103 \mathrm{MA}$ ) containing $1 \mathrm{x}$ protease inhibitor cocktail, centrifuged at $13,000 \mathrm{~g}$ for 5 minutes at $4^{\circ} \mathrm{C}$ to 104 remove cell debris, and frozen at $-80^{\circ} \mathrm{C}$ overnight. Protein concentrations were then quantified 105 using a BCA protein detection kit (Thermo Scientific, Waltham, MA). Caspase activities were 106 determined using fluorogenic caspase substrates selective for either caspase-3 (5 $\mu \mathrm{M}$

107 Ac-DEVD-AFC), caspase-9 (10 $\mu$ M Ac-LEHD-AFC) or caspase-8 (10 $\mu \mathrm{M}$ Ac-IETD-AFC) in 10 $108 \mu \mathrm{g}$ of extracted protein suspended in caspase reaction buffer (20 mM PIPES at pH 7.2, $30 \mathrm{mM}$ $109 \mathrm{NaCl}, 10 \mathrm{mM}$ DTT, 1 mM EDTA, 0.1\% CHAPS, 10\% sucrose). The time-dependent release of 110 7-amino-4-trifluoromethyl coumarin (AFC) was measured using a SpectroMax M5 microplate 111 reader (Molecular Devices, Sunnydale, CA) at an excitation wavelength of $400 \mathrm{~nm}$ and an 112 emission wavelength of $505 \mathrm{~nm}$. Measurements were recorded at 2 minute intervals for 90 113 minutes. A standard curve of AFC fluorescence was used to calculate the amount of AFC 114 released (in picomoles) during each reaction.

SDS-PAGE and Western Blot.

$116 \quad$ Crude protein extracts $(50 \mu \mathrm{g})$ were resolved by electrophoresis using $10 \%$ sodium dodecyl 117 sulfate-polyacrylamide gels and then transferred to a PVDF Immobilon-P membrane (Bio-Rad, 118 Mississauga, ON). Blots were blocked using 5\% milk powder (Selection brand, Marché 119 Jean-Talon, Montréal, QC) and incubated with antibodies as follows: 1:250 dilution for 120 anti-caspase-3, 1:1000 for anti-cleaved PARP, 1:1000 for anti-Bcl-2, 1:1000 for anti-Bax, and 121 1:1000 for anti-Bid. Immunoreactive proteins were exposed to anti-rabbit horseradish 122 peroxidise-conjugated secondary antibodies (Millipore) that were diluted 1:5000.

123 Antigen-antibody complexes were detected using Immobilon ECL Western Chemiluminescent

124 HRP Substrate (Millipore) and recorded with a Versadoc imaging system (Bio-Rad). Total

125 protein content per well was determined using 1x Amido Black staining solution

126 (Sigma-Aldrich).

127 Mass Spectrometry

128 Mass spectrometry-based analysis of LCA and UDCA was performed as previously reported . In 129 brief, lipids were extracted by a modified Bligh and Dyer method from cells pelleted by 130 centrifugation for $5 \mathrm{~min}$ at $16,000 \times \mathrm{g}$ at $4^{\circ} \mathrm{C}$ and from the supernatant of cultural medium. The 131 extracted lipids were dried under nitrogen and resuspended in chloroform. Immediately prior to 132 injection the extracted lipids were combined with a 2:1 methanol:chloroform mixture 
133 supplemented with $0.1 \%(\mathrm{v} / \mathrm{v})$ ammonium hydroxide. The sample was injected directly into a 134 Thermo Orbitrap Velos equipped with a HESI-II ion source (Thermo Scientific, Waltham, MA, $135 \mathrm{USA}$ ) at a flow rate of $5 \mu \mathrm{l} / \mathrm{min}$. Spectra were obtained in negative-ion mode. The source voltage 136 was set to $4.0 \mathrm{kV}$, a capillary temperature of $275^{\circ} \mathrm{C}$, a sheath gas flow of 5 (arbitrary units) and 137 an auxiliary gas flow of 1 (arbitrary units). Acquired spectra were exported from Xcalibur 138 software (Thermo Scientific) and then deconvoluted and deisotoped using Excel Macros.

139 Statistical Analysis.

140 All experiments were performed in at least triplicate using cells after various passages and 141 the data are presented as mean \pm SEM. Statistically significant differences $(\mathrm{p}<0.05)$ between 142 various treatments and untreated cells were determined using a two-tailed Student t-test with 143 Bonferroni correction for multiple comparisons. IC $_{50}$ values for inhibition of cell viability were 144 calculated using a sigmoidal curve-fitting model of log-inhibitor concentration versus normalized 145 inhibition response, with variable slope (GraphPad Prism v5.03, GraphPad Software, San Diego, 146 CA).

\section{Results}

149 Bile acids inhibit proliferation and induce cell death in LNCaP and PC-3 cells.

A 48 hour treatment with LCA significantly decreased the number of intact LNCaP and PC-3 cells, with $\mathrm{IC}_{50}$ values of $40.5 \pm 0.07 \mu \mathrm{M}$ and $74.9 \pm 0.25 \mu \mathrm{M}$, respectively, without decreasing the viability of non-tumorigenic RWPE-1 cells (Fig. 1A). The hydrophobic bile acids DCA and CDCA were less cytotoxic than LCA, decreasing cell viability at concentrations above $100 \mu \mathrm{M}$ in LNCaP and PC-3 cells (Fig. 1B, 1C). Relatively hydrophilic bile acids, such as HDCA and UDCA decreased the number of intact cells at concentrations above $300 \mu \mathrm{M}$ in either cell line, whereas CA was not cytotoxic at concentrations as high as $500 \mu \mathrm{M}$.

In addition to LCA-mediated inhibition of cell viability, we assessed the ability of lower concentrations of LCA to inhibit the AD proliferation of AR positive $\mathrm{LNCaP}$ prostate cancer cells when stimulated with DHT. Indeed, LCA decreased the proliferation of androgen-stimulated LNCaP cells in a concentration-dependent manner with an IC $\mathrm{C}_{50}$ of $8.5 \mu \mathrm{M} \pm$ 1.9 (Fig. 1D). 
162

163

164

165

166

167

168

169

170

171

172

173

174

175

176

177

178

179

180

181

182

183

184

185

186

187

188

189

190

LCA induces a caspase-3-dependent apoptotic programme.

To determine whether the caspases play a role in bile acid-induced prostate cancer cell death, we determined the effects of LCA on caspase-3 activity in AD LNCaP and AI PC-3 cells. LNCaP and PC-3 cells exposed to sub-cytotoxic and cytotoxic concentrations of LCA for 24 hours contained increased levels of the cleaved and active 17 and $20 \mathrm{kDa}$ subunits of the $34 \mathrm{KDa}$ caspase-3 zymogen (Fig. 2A). In concordance with this observation, the catalytic activity of caspase-3 was also increased after exposure to (sub)cytotoxic concentrations of LCA (Fig. 2B). Also, levels of the $89 \mathrm{kDa}$ fragment of poly ADP ribose polymerase (PARP), an endogenous substrate of caspase-3 usually cleaved during apoptosis, were significantly elevated in LNCaP cells, but not in PC-3 cells (Fig. 2C). Moreover, a cell permeable inhibitor of caspase-3, z-DEVD-fmk, partially inhibited LCA-induced cell death in both cell lines (Fig. 2D).

LCA does not accumulate inside LNCaP or PC-3 cells.

To determine the extent to which LCA was able to enter human prostate cancer cells, we determined the intra/extra cellular distribution of LCA under our experimental cell culture conditions. LNCaP and PC-3 cells did not accumulate LCA, with as much as 98\% of the nominal LCA concentrations present in the extracellular medium of LNCaP and PC-3 cultures after 24 hours (Table 1). Also, neither cell line was able to accumulate the relatively hydrophilic bile acid, UDCA, when treated with concentrations as high as $75 \mu \mathrm{M}$ for 24 hours (Table 1).

LCA activates extrinsic and intrinsic pathways of apoptosis in human prostate cancer cell lines.

The inability of LCA to significantly accumulate inside prostate cancer cells led us to explore if LCA-induced cell death may occur through activation of the extrinsic pathway of apoptosis. We found increased levels of active caspase-8 in extracts of both LNCaP and PC-3 cells treated with increasing concentrations of LCA (Fig. 3A). LCA-induced cell toxicity was also alleviated in the presence of the caspase-8 inhibitor, z-IETD-fmk (Fig. 3B). Moreover, we found statistically significant increases of caspase-9 activity in both cell lines (Fig. 4A) in addition to cleavage of pro-apoptotic Bcl-2 related proteins Bax and Bid (Fig 4B). However, we found decreased levels of Bcl-2 in PC-3 cells only (Fig. 4B). We observed only a slight decrease in MMP in LNCaP cells after 8 hours of exposure to LCA, but in PC-3 cells we observed a marked decrease in MMP as early as after 1 hour of treatment with LCA (Fig. 4C). 


\section{Discussion}

193 We have previously shown that LCA can selectively kill human neuroblastoma cells at 194 concentrations non-toxic to normal human primary neurons . In the present study, we provide 195 evidence that LCA also possesses selective anticancer properties against cultured AD and AI 196 prostate cancer cells, whilst not affecting the viability of normal epithelial prostate cells.

197 LCA activates a caspase-dependent mode of apoptosis in AD and AI prostate cancer cells.

198 We have shown that LCA kills LNCaP and PC-3 cells in a caspase-dependent manner by 199 activating the intrinsic and extrinsic pathways of apoptosis. In both prostate cancer cell types, 200 cell death induced by LCA appears to be at least partly dependent on the activity of initiator 201 caspase-8. Our observation is similar to those made in studies where treatment of hepatocytes 202 and colon cancer cells with bile acids resulted in a TGR5-dependent increase in levels of 203 CD95/Fas death receptor in the plasma membrane, facilitating the activation of caspase-8 and its 204 downstream apoptotic machinery . TGR5 is a cell surface membrane-bound metabotropic 205 G-protein-coupled receptor that is highly conserved among species and is found predominantly 206 in gall bladder and intestinal epithelium . Although TGR5 mRNA has been found in prostate 207 cells, its function in this tissue has yet to be established. LCA is a potent natural agonist of 208 TGR5 and, upon direct binding to the receptor, activates a cAMP/PKA signalling cascade, 209 resulting in the modification the oxidation-reduction processes in the mitochondria with resultant 210 increase in the generation of reactive oxygen species, thereby promoting the vesicle-mediated 211 trafficking of CD95/Fas from the Golgi apparatus to the plasma membrane . Interaction between

212 LCA and TGR5 may also stimulate the phosphorylation of c-Jun-N terminal kinase (JNK)

213 through activation of the MEKKK1/2/3-MKK4/7 pathway, resulting in the release of

214 pro-caspase-8 from JNK and enabling its recruitment to CD95/Fas . That LCA does not

215 accumulate inside either LNCaP or PC-3 cells implies that LCA interacts directly with a cell 216 surface receptor in order to activate the extrinsic pathway of apoptosis, and that this identity of

217 this receptor is likely to be TGR5 as it is the only known cell surface receptor that binds bile 218 acids, a hypothesis we are currently investigating in detail.

219 Our observation that Bid is cleaved after treatment with LCA suggests that once caspase-8 220 is activated it would continue to cleave Bid, thereby initiating the intrinsic pathway of apoptosis. 
221 In fact, treatment of neuroblastoma cells with LCA resulted in MOMP, which allowed the exit of 222 cytochrome c, formation of the apoptosome and ultimately activation of caspase-9 . In the 223 present study, we show that LCA induces Bax cleavage, suggesting an induction of MOMP and 224 mitochondrial fragmentation, resulting in the observed activation of caspase-9 in both cell lines.

225 Moreover, we show that LCA causes loss of MMP in PC-3 cells as soon as 1 hour after 226 treatment, showing that LCA induces MOMP in at least one type of prostate cancer cell, in 227 addition to neuroblastoma cells, and that the induction of MOMP is an early-stage event in the 228 induction of apoptosis in these cells. We did not observe a statistically significant decrease in 229 MMP in LNCaP cells up to 8 hours after exposure to LCA, indicating that the onset of MOMP in 230 these cells is a later-stage event that would appear to occur after activation of caspase-8. 231 Therefore, it is possible that LCA may transmit a MOMP-activating signal through interaction 232 with a cell surface receptor that requires numerous steps in order to target the mitochondria and 233 activate the intrinsic pathway of apoptosis. Additionally, the reduction in the levels of Bcl-2 234 observed only in PC-3 cells may suggest a larger role for Bcl-2 in promoting the intrinsic 235 pathway of apoptosis in these cells, and may help explain why LCA increased MOMP much 236 earlier in PC-3 cells than in LNCaP cells.

We saw increases in the activity of caspase-3 in each cell line treated with LCA, and cell death induced by LCA was only partially dependent on this main effector caspase. Previous studies have shown that the cleaved form of Bax can significantly sensitize cells to stress-induced apoptosis by releasing cytochrome c, apoptosis-inducing factor and endonuclease

241 G from the mitochondria . Paired with our observation that inhibition of caspase-8 did not completely abrogate LCA-induced cell death, it is likely that LCA induces both caspase-dependent and -independent modes of apoptosis in prostate cancer cells.

Pharmacophore modeling of the anticancer activity of bile acids.

We tested the ability of a wide range of bile acids to induce cell death in AD (LNCaP) and 246 AI (PC-3) prostate cancer cells and found that LCA was the most effective bile acid, while two other hydrophobic bile acids, DCA and CDCA, were moderately toxic to these cells. Addition of alpha-oriented hydroxyl groups at the 7- or 12-positions (CDCA and DCA, respectively) or at the 6-position (HDCA) significantly reduced the cytotoxicity of the bile acid structure. Moreover, addition of beta-oriented hydroxyl groups to the molecule at the 12-position (UDCA) 
251 further reduced the toxicity of the bile acid in each cell line, whereas the addition of two

252 alpha-oriented hydroxyl groups to both the 7- and 12-positions rendered the resultant bile acids

253 non-cytotoxic. Therefore, reduction of the hydrophobicity of the alpha- or beta-faces of the

254 steroid backbone is sufficient to negate the toxicity of the molecule. Such a relationship between

255 bile acid hydrophobicity and potency, where the hydrophobicity is directly correlated with the

256 biological activity of the molecule, has been described in two other contexts: 1) TGR5 receptor

257 activation in a reporter system , and 2) extension of lifespan of chronologically aging yeast . In

258 support of a possible involvement of TGR5 in bile acid-induced cell death, we found a highly

259 significant correlation between the potency of each cytotoxic bile acid in LNCaP cells and its

260 reported $\mathrm{EC}_{50}$ for induction of TGR5 receptor-mediated luciferase activity ( $\mathrm{r}=0.96 ; \mathrm{n}=4$;

$261 \mathrm{p}<0.001)$ in transiently transfected Chinese Ovarian Hamster cells . The inability of LCA and

262 UDCA to enter LNCaP or PC-3 cells would suggest they would have equal opportunity to

263 interact with a cell surface membrane receptor in order to induce apoptosis, yet LCA was far

264 more potent than UDCA, supporting the notion that a specific interaction between LCA and a

265 cell surface receptor, possibly TGR5, could be the event responsible for the apoptotic death of

266 prostate cancer cells. With respect to the lesser toxic bile acids, our results are consistent with a

267 previous study of bile acids in PC-3 cells where concentrations of CDCA and UDCA as high as

$268100 \mu \mathrm{M}$ did not result in significant cell death .

269

LCA inhibits proliferation of $A D$ prostate cancer cells.

270

271

In our study LCA inhibits the proliferation of DHT-stimulated LNCaP cells, yet it is unlikely that LCA directly antagonizes the AR, because it does not accumulate inside LNCaP

272 cells. Instead, it is possible that the inhibition of LNCaP cell growth is related to the ability of

273 LCA to interact with cell surface receptors, such as TGR5, which can activate JNK, thereby

274 antagonizing the NFKB pro-survival pathway, or any other cell surface receptor capable of

275 inhibiting androgen-independent processes related to proliferation of these cells. It has

276 previously been reported that LCA can directly bind to two key negative regulators of p53,

277 MDM2 and MDM4 . However, it is unlikely that LCA directly inhibits either of these

278 cytoplasmic proteins directly due to its inability to enter prostate cancer cells. It is still possible

279 that LCA might upregulate p53 expression via a Mnt-Max to Myc-Max switch in nuclear binding

280 of E-box sequences in these cells, but our results imply that this would be more likely the result

281 of an upstream event, such as the activation of a cell surface receptor. 
283 Our findings in this report share several similarities with those we described previously in 284 neuroblastoma cells treated with LCA : 1) LCA elicits apoptosis in a caspase-3 dependent 285 manner, 2) LCA activates both extrinsic and intrinsic pathways of apoptosis and 3) LCA enters 286 neither neuroblastoma nor prostate cancer cells. It is then probable that these cancerous tissues 287 share a common target, which is most likely localized at the surface of the plasma membrane and 288 is either activated or deactivated by LCA in order to elicit apoptosis. Additionally, the 289 concentrations used to kill both prostate cancer was found to be in a similar range to that of 290 neuroblastoma cells (between 25 and $100 \mu \mathrm{M}$ ), and these concentrations were found to be 291 non-toxic to both normal human primary neurons and non-tumourigenic immortalised prostate 292 cells. We have also found that concentrations well below those needed to induce apoptosis $293(2.5-10 \mu \mathrm{M})$ can inhibit the proliferation of AD prostate cancer cells. Though ingesting amounts 294 of LCA in order to reach plasma concentrations as high as these might be lethal, methods of 295 employing LCA in a more targeted manner, using nanoparticle-encapsulation techniques or 296 delivery via infection with the bacteria Listeria monocytogenes, allowing for its accumulation in 297 only immune-compromised metastatic tissues without killing the surrounding tissues, could be 298 successfully developed to employ LCA as an anti-cancer compound. It is then pertinent to 299 understand the exact molecular mechanism of cell death instigated by LCA, to develop novel 300 strategies to enhance the ability of LCA or newly designed compounds to trigger (the)

301 LCA-mediated anticancer pathway(s), as well as to validate these strategies using in vivo models 302 of neuroblastoma, prostate and other cancers. 
305 Table 1. Extra/intracellular distribution of LCA and UDCA in LNCaP and PC-3 human prostate 306 cancer cells in culture. Cells were separated from cultural media and the compounds were

307 extracted and their concentrations measured by mass spectrometry as described in Materials and 308 Methods. Percentages are presented as means \pm SD of three independent experiments.

\begin{tabular}{llccc}
\hline \multirow{2}{*}{ Cell Line } & \multirow{2}{*}{ Compound } & $\begin{array}{c}\text { Concentration } \\
(\mu \mathrm{M})\end{array}$ & \multicolumn{2}{c}{ \% of Compound Recovered } \\
\cline { 3 - 5 } & & 25 & $97.28 \pm 1.10$ & $2.72 \pm 1.10$ \\
\cline { 3 - 5 } LNCaP & LCA & 50 & $94.15 \pm 2.45$ & $5.85 \pm 2.45$ \\
& & 25 & $90.40 \pm 3.86$ & $9.60 \pm 3.86$ \\
& \multirow{2}{*}{ UDCA } & 50 & $95.77 \pm 0.10$ & $4.23 \pm 0.10$ \\
\hline \multirow{2}{*}{ PC-3 } & LCA & 50 & $97.91 \pm 0.05$ & $2.09 \pm 0.05$ \\
& & 75 & $97.61 \pm 1.93$ & $2.39 \pm 1.93$ \\
\cline { 2 - 5 } & \multirow{2}{*}{ UDCA } & 50 & $97.56 \pm 0.17$ & $2.44 \pm 0.17$ \\
& & 75 & $97.57 \pm 0.67$ & $2.43 \pm 0.67$ \\
\hline
\end{tabular}

Figure legends

311 Figure 1. Bile acids inhibit proliferation and induce apoptosis in androgen-dependent LNCaP 312 and -independent PC-3 prostate cancer cells. (A) Percentage of intact LNCaP, PC-3 and RWPE-1 313 cells that did not have fragmented nuclei (apoptotic), condensed chromatin (apoptotic), or 314 propidium iodide staining (necrotic) was calculated 48 hours after treatment with 50 or $75 \mu \mathrm{M}$ of 315 lithocholic acid (LCA). The percentage of intact LNCaP cells (B) and PC-3 cells (C) was

316 calculated 48 hours after treatment with increasing concentrations $(10-500 \mu \mathrm{M})$ of lithocholic 317 (LCA, •), deoxycholic (DCA, $\bullet$ ), chenodeoxycholic (CDCA, $\square$ ), hyodeoxycholic (HDCA, $\mathbf{\Delta}$ ), 318 ursodeoxycholic (UDCA, $\Delta$ ) or cholic (CA, ○) acid. (D) Relative androgen-dependent growth 319 rates of LNCaP cells grown in stripped RPMI 1640 medium without phenol-red and co-treated 320 with $0.1 \mathrm{nM}$ DHT and increasing concentrations (1-25 $\mu \mathrm{M})$ of LCA. Data are presented as means $321 \pm \operatorname{SEM}(n=3-5)$. 
322 Figure 2. LCA-induced cell death is a caspase-3-dependent process. Cleavage of caspase-3

323 protein was assessed by western blot (A) and catalytic activity (B) was measured by cleavage of

324 the fluorogenic substrate Ac-DEVD-AFC in response to a 24 hour treatment of LNCaP cells and 325 PC-3 cells with increasing concentrations (25-75 $\mu \mathrm{M}$ ) of LCA. (C) Cleavage of PARP after 24 326 hour exposure of LNCaP cells to increasing concentrations (25-75 $\mu \mathrm{M})$ of LCA. (D) Inhibition 327 of cell death after a 24 hour co-exposure of LNCaP $(40 \mu \mathrm{M})$ or PC-3 $(50 \mu \mathrm{M})$ cells to LCA and $32810 \mu \mathrm{M}$ of the membrane permeable caspase-3 inhibitor z-DEVD-fmk. In (B) and (D) responses 329 are presented as means \pm SEM $(\mathrm{n}=3-5) ;{ }^{*} \mathrm{p}<0.05 ;{ }^{* * *} \mathrm{p}<0.001$.

330 Figure 3. LCA activates the extrinsic pathway of apoptosis in androgen-dependent and 331 -independent prostate cancer cells. (A) Activity of caspase-8 was measured by cleavage of the 332 fluorogenic substrate Ac-IETD-AFC after 24 hours of treatment of LNCaP and PC-3 cells with 333 increasing concentrations (25-75 $\mu \mathrm{M}$ ) of LCA. (B) Inhibition of cell death after a 24 hour 334 co-exposure of LNCaP $(40 \mu \mathrm{M})$ or PC-3 $(50 \mu \mathrm{M})$ cells to LCA and $10 \mu \mathrm{M}$ of the membrane 335 permeable caspase-8 inhibitor z-IETD-fmk. Activities are presented as means \pm SEM $(n=3-5)$; 336 ${ }^{*} \mathrm{p}<0.05 ;{ }^{* *} \mathrm{p}<0.01$.

337 338

Figure 4. LCA activates the intrinsic pathway of apoptosis in androgen-dependent and -independent prostate cancer cells. (A) Activity of caspase-9 was measured by cleavage of the fluorogenic substrate Ac-LEHD-AFC after a 24 hour treatment of LNCaP and PC-3 cells with increasing concentrations (25-75 $\mu \mathrm{M}$ ) of LCA. (B) Expression levels of Bcl-2 and cleavage of Bax and Bid after a $24 \mathrm{~h}$ exposure of LNCaP and PC-3 cells to increasing concentrations (25-75 $\mu \mathrm{M}$ ) of LCA. (C) Mitochondrial membrane permeability was measured using TMRE in LNCaP and PC-3 cells treated with 50 and $75 \mu \mathrm{M}$ LCA, respectively. In (A) and (C) responses are presented as means \pm SEM $(n=3-5) ;{ }^{*}<0.05 ;{ }^{* *} \mathrm{p}<0.01$.

\section{References}

Baxter, J. D. \& Webb, P. 2006. Metabolism: bile acids heat things up. Nature, 439, 402-3.

Bourque, S. D. \& Titorenko, V. I. 2009. A quantitative assessment of the yeast lipidome using electrospray ionization mass spectrometry. $J$ Vis Exp.

Cabon, L., Galan-Malo, P., Bouharrour, A., Delavallee, L., Brunelle-Navas, M. N., Lorenzo, H. K., et al. 2012. BID regulates AIF-mediated caspase-independent necroptosis by promoting BAX activation. Cell Death Differ, 19, 245-56. 
353

354

355

356

357

358

359

360

361

362

363

364

365

366

367

368

369

370

371

372

373

374

375

376

377

378

379

380

381

382

383

384

385

386

387

388

389

390

391

392

393

394

395

396

397

Cao, X., Deng, X. \& May, W. S. 2003. Cleavage of Bax to p18 Bax accelerates stress-induced apoptosis, and a cathepsin-like protease may rapidly degrade p18 Bax. Blood, 102, 2605-14.

Chen, X., Lou, G., Meng, Z. \& Huang, W. 2011. TGR5: a novel target for weight maintenance and glucose metabolism. Exp Diabetes Res, 2011, 853501.

Choi, Y. H., Im, E. O., Suh, H., Jin, Y., Yoo, Y. H. \& Kim, N. D. 2003. Apoptosis and modulation of cell cycle control by synthetic derivatives of ursodeoxycholic acid and chenodeoxycholic acid in human prostate cancer cells. Cancer Lett, 199, 157-67.

Foord, S. M., Bonner, T. I., Neubig, R. R., Rosser, E. M., Pin, J. P., Davenport, A. P., et al. 2005. International Union of Pharmacology. XLVI. G protein-coupled receptor list. Pharmacol Rev, 57, 279-88.

Gao, G. \& Dou, Q. P. 2000. N-terminal cleavage of bax by calpain generates a potent proapoptotic 18-kDa fragment that promotes bcl-2-independent cytochrome $\mathrm{C}$ release and apoptotic cell death. J Cell Biochem, 80, 53-72.

Giordano, C., Catalano, S., Panza, S., Vizza, D., Barone, I., Bonofiglio, D., et al. 2011. Farnesoid $X$ receptor inhibits tamoxifen-resistant MCF-7 breast cancer cell growth through downregulation of HER2 expression. Oncogene, 30, 4129-40.

Goldberg, A. A., Beach, A., Davies, G. F., Harkness, T. A., Leblanc, A. \& Titorenko, V. I. 2011. Lithocholic bile acid selectively kills neuroblastoma cells, while sparing normal neuronal cells. Oncotarget, 2, 761-82.

Goldberg, A. A., Richard, V. R., Kyryakov, P., Bourque, S. D., Beach, A., Burstein, M. T., et al. 2010. Chemical genetic screen identifies lithocholic acid as an anti-aging compound that extends yeast chronological life span in a TOR-independent manner, by modulating housekeeping longevity assurance processes. Aging (Albany NY), 2, 393-414.

Hageman, J., Herrema, H., Groen, A. K. \& Kuipers, F. 2010. A role of the bile salt receptor FXR in atherosclerosis. Arterioscler Thromb Vasc Biol, 30, 1519-28.

Hylemon, P. B., Zhou, H., Pandak, W. M., Ren, S., Gil, G. \& Dent, P. 2009. Bile acids as regulatory molecules. J Lipid Res, 50, 1509-20.

Katona, B. W., Anant, S., Covey, D. F. \& Stenson, W. F. 2009. Characterization of enantiomeric bile acid-induced apoptosis in colon cancer cell lines. J Biol Chem, 284, 3354-64.

Kawamata, Y., Fujii, R., Hosoya, M., Harada, M., Yoshida, H., Miwa, M., et al. 2003. A G protein-coupled receptor responsive to bile acids. J Biol Chem, 278, 9435-40.

Kim, N. D., Im, E., Yoo, Y. H. \& Choi, Y. H. 2006. Modulation of the cell cycle and induction of apoptosis in human cancer cells by synthetic bile acids. Curr Cancer Drug Targets, 6, 681-9.

Lefebvre, P., Cariou, B., Lien, F., Kuipers, F. \& Staels, B. 2009. Role of bile acids and bile acid receptors in metabolic regulation. Physiol Rev, 89, 147-91.

Lim, S. C., Duong, H. Q., Parajuli, K. R. \& Han, S. I. 2012. Pro-apoptotic role of the MEK/ERK pathway in ursodeoxycholic acid-induced apoptosis in SNU601 gastric cancer cells. Oncol Rep, 28, 1429-34.

Makadia, H. K. \& Siegel, S. J. 2011. Poly Lactic-co-Glycolic Acid (PLGA) as Biodegradable Controlled Drug Delivery Carrier. Polymers (Basel), 3, 1377-1397. 
398

399

400

401

402

403

404

405

406

407

408

409

410

411

412

413

414

415

416

417

418

419

420

421

422

423

424

425

426

427

428

429

430

431

432

433

434

435

436

437

438

439

440

441

442

McLeod, D. G. 1997. Tolerability of Nonsteroidal Antiandrogens in the Treatment of Advanced Prostate Cancer. Oncologist, 2, 18-27.

Merrill, R. A., Dagda, R. K., Dickey, A. S., Cribbs, J. T., Green, S. H., Usachev, Y. M., et al. 2011. Mechanism of neuroprotective mitochondrial remodeling by PKA/AKAP1. PLoS Biol, 9, e1000612.

Moubarak, R. S., Yuste, V. J., Artus, C., Bouharrour, A., Greer, P. A., Menissier-de Murcia, J., et al. 2007. Sequential activation of poly(ADP-ribose) polymerase 1, calpains, and Bax is essential in apoptosis-inducing factor-mediated programmed necrosis. Mol Cell Biol, 27, 4844-62.

Nunes, A. F., Amaral, J. D., Lo, A. C., Fonseca, M. B., Viana, R. J., Callaerts-Vegh, Z., et al. 2012. TUDCA, a bile acid, attenuates amyloid precursor protein processing and amyloid-beta deposition in APP/PS1 mice. Mol Neurobiol, 45, 440-54.

Peiro-Jordan, R., Krishna-Subramanian, S., Hanski, M. L., Luscher-Firzlaff, J., Zeitz, M. \& Hanski, C. 2012. The chemopreventive agent ursodeoxycholic acid inhibits proliferation of colon carcinoma cells by suppressing c-Myc expression. Eur J Cancer Prev, 21, 413-22.

Pols, T. W., Noriega, L. G., Nomura, M., Auwerx, J. \& Schoonjans, K. 2011. The bile acid membrane receptor TGR5 as an emerging target in metabolism and inflammation. J Hepatol, 54, 1263-72.

Quispe-Tintaya, W., Chandra, D., Jahangir, A., Harris, M., Casadevall, A., Dadachova, E., et al. 2013. Nontoxic radioactive Listeriaat is a highly effective therapy against metastatic pancreatic cancer. Proc Natl Acad Sci U S A.

Ramalho, R. M., Viana, R. J., Low, W. C., Steer, C. J. \& Rodrigues, C. M. 2008. Bile acids and apoptosis modulation: an emerging role in experimental Alzheimer's disease. Trends Mol Med, 14, 54-62.

Sato, H., Macchiarulo, A., Thomas, C., Gioiello, A., Une, M., Hofmann, A. F., et al. 2008. Novel potent and selective bile acid derivatives as TGR5 agonists: biological screening, structure-activity relationships, and molecular modeling studies. $J$ Med Chem, 51, 1831-41.

Schneider, C. A., Rasband, W. S. \& Eliceiri, K. W. 2012. NIH Image to ImageJ: 25 years of image analysis. Nat Methods, 9, 671-5.

Siegel, R., Naishadham, D. \& Jemal, A. 2012. Cancer statistics, 2012. CA: A Cancer Journal for Clinicians, 62, 10-29.

Sodeman, T., Bronk, S. F., Roberts, P. J., Miyoshi, H. \& Gores, G. J. 2000. Bile salts mediate hepatocyte apoptosis by increasing cell surface trafficking of Fas. Am J Physiol Gastrointest Liver Physiol, 278, G992-9.

Sola, S., Castro, R. E., Laires, P. A., Steer, C. J. \& Rodrigues, C. M. 2003. Tauroursodeoxycholic acid prevents amyloid-beta peptide-induced neuronal death via a phosphatidylinositol 3-kinase-dependent signaling pathway. Mol Med, 9, 226-34.

Thomas, C., Gioiello, A., Noriega, L., Strehle, A., Oury, J., Rizzo, G., et al. 2009. TGR5-mediated bile acid sensing controls glucose homeostasis. Cell Metab, 10, 167-77.

Thomas, C., Pellicciari, R., Pruzanski, M., Auwerx, J. \& Schoonjans, K. 2008. Targeting bile-acid signalling for metabolic diseases. Nat Rev Drug Discov, 7, 678-93. 
443

444

445

446

447

448

449

450

451

452

453

454

455

456

457

458

459

460

461

462

463

464

465

466

467

468

469

470

471

472

473

474

475

476

477

478

479

480

481

Tiwari, A. \& Maiti, P. 2009. TGR5: an emerging bile acid G-protein-coupled receptor target for the potential treatment of metabolic disorders. Drug Discov Today, 14, 523-30.

Toyota, H., Yanase, N., Yoshimoto, T., Moriyama, M., Sudo, T. \& Mizuguchi, J. 2003. Calpain-induced Bax-cleavage product is a more potent inducer of apoptotic cell death than wild-type Bax. Cancer Lett, 189, 221-30.

Trauner, M., Claudel, T., Fickert, P., Moustafa, T. \& Wagner, M. 2010. Bile acids as regulators of hepatic lipid and glucose metabolism. Dig Dis, 28, 220-4.

Vallim, T. Q. \& Edwards, P. A. 2009. Bile acids have the gall to function as hormones. Cell Metab, 10, 162-4.

Viana, R. J., Nunes, A. F., Castro, R. E., Ramalho, R. M., Meyerson, J., Fossati, S., et al. 2009. Tauroursodeoxycholic acid prevents E22Q Alzheimer's Abeta toxicity in human cerebral endothelial cells. Cell Mol Life Sci, 66, 1094-104.

Vogel, S. M., Bauer, M. R., Joerger, A. C., Wilcken, R., Brandt, T., Veprintsev, D. B., et al. 2012. Lithocholic acid is an endogenous inhibitor of MDM4 and MDM2. Proc Natl Acad Sci U S A, 109, 16906-10.

Wachs, F. P., Krieg, R. C., Rodrigues, C. M., Messmann, H., Kullmann, F., Knuchel-Clarke, R., et al. 2005. Bile salt-induced apoptosis in human colon cancer cell lines involves the mitochondrial transmembrane potential but not the CD95 (Fas/Apo-1) receptor. Int J Colorectal Dis, 20, 103-13.

Whiteman, M., Chu, S. H., Siau, J. L., Rose, P., Sabapathy, K., Schantz, J. T., et al. 2007. The pro-inflammatory oxidant hypochlorous acid induces Bax-dependent mitochondrial permeabilisation and cell death through AIF-/EndoG-dependent pathways. Cell Signal, 19, 705-14.

Wood, D. E. \& Newcomb, E. W. 2000. Cleavage of Bax enhances its cell death function. Exp Cell Res, 256, 375-82.

Wysowski, D. K., Freiman, J. P., Tourtelot, J. B. \& Horton, M. L., 3rd 1993. Fatal and nonfatal hepatotoxicity associated with flutamide. Ann Intern Med, 118, 860-4.

Yang, H., Li, T. W., Ko, K. S., Xia, M. \& Lu, S. C. 2009. Switch from Mnt-Max to Myc-Max induces p53 and cyclin D1 expression and apoptosis during cholestasis in mouse and human hepatocytes. Hepatology, 49, 860-70.

Yang, J. I., Yoon, J. H., Myung, S. J., Gwak, G. Y., Kim, W., Chung, G. E., et al. 2007. Bile acid-induced TGR5-dependent C-Jun- $N$ terminal kinase activation leads to enhanced caspase 8 activation in hepatocytes. Biochem Biophys Res Commun, 361, 156-61.

Zhong, M. 2010. TGR5 as a therapeutic target for treating obesity. Curr Top Med Chem, 10, 386-96. 


\section{Figure 1}

Bile acids inhibit proliferation and induce apoptosis in androgen-dependent LNCaP and -independent PC-3 prostate cancer cells.

(A) Percentage of intact LNCaP, PC-3 and RWPE-1 cells that did not have fragmented nuclei (apoptotic), condensed chromatin (apoptotic), or propidium iodide staining (necrotic) was calculated 48 hours after treatment with 50 or $75 \mu \mathrm{M}$ of lithocholic acid (LCA) . The percentage of intact LNCaP cells (B) and PC-3 cells (C) was calculated 48 hours after treatment with increasing concentrations (10-500 $\mu \mathrm{M}$ ) of lithocholic ( LCA, •), deoxycholic (DCA, $\square$ ), chenodeoxycholic ( CDCA, $\square$ ), hyodeoxycholic (HDCA, $\boldsymbol{\Delta}$ ), ursodeoxycholic (UDCA, $\Delta$ ) or cholic (CA, ○) acid. (D) Relative androgen-dependent growth rates of LNCaP cells grown in stripped RPMI 1640 medium without phenol-red and co-treated with $0.1 \mathrm{nM}$ DHT and increasing concentrations (1-25 $\mu \mathrm{M})$ of LCA. Data are presented as means $\pm \operatorname{SEM}(n=3-5)$. 
A

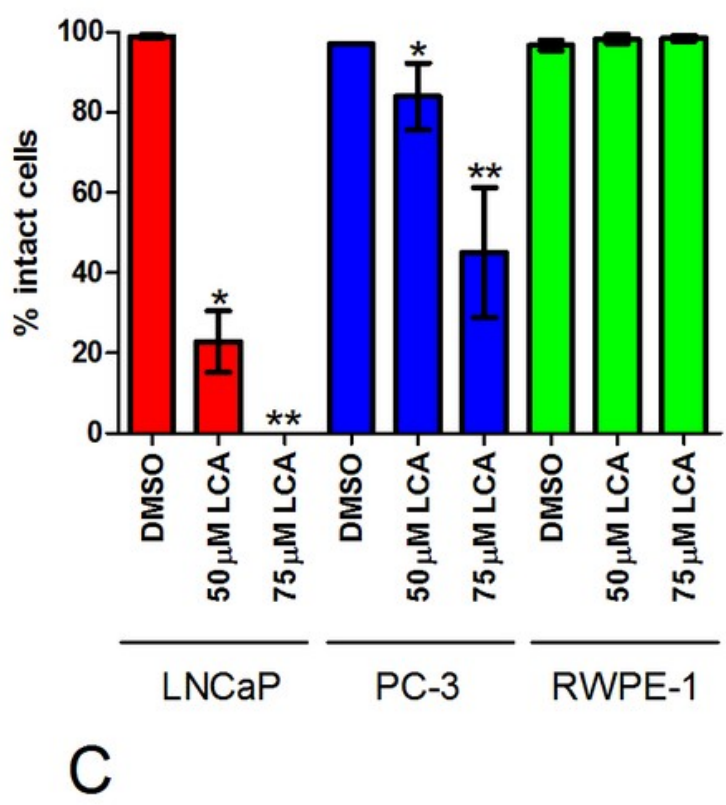

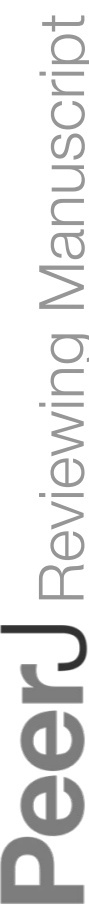

B

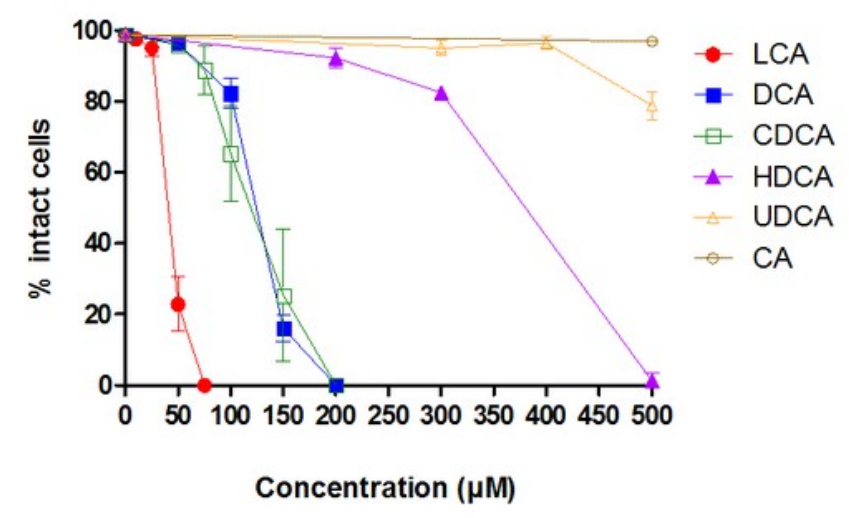

D

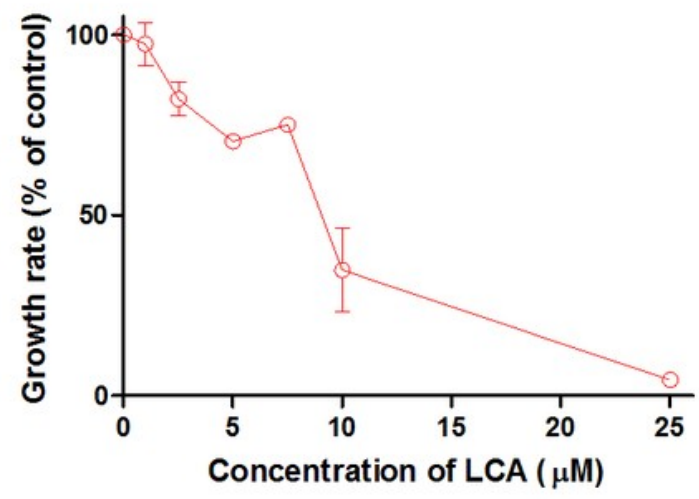




\section{Figure 2}

LCA-induced cell death is a caspase-3-dependent process.

Cleavage of caspase-3 protein was assessed by western blot (A) and catalytic activity (B) was measured by cleavage of the fluorogenic substrate Ac-DEVD-AFC in response to a 24 hour treatment of LNCaP cells and PC-3 cells with increasing concentrations (25-75 $\mu \mathrm{M})$ of LCA. ( C) Cleavage of PARP after 24 hour exposure of LNCaP cells to increasing concentrations (25-75 $\mu \mathrm{M})$ of LCA. (D) I nhibition of cell death after a 24 hour co-exposure of LNCaP $(40 \mu \mathrm{M})$ or PC-3 $(50 \mu \mathrm{M})$ cells to LCA and $10 \mu \mathrm{M}$ of the membrane permeable caspase-3 inhibitor z-DEVD-fmk. In (B) and (D) responses are presented as means $\pm \operatorname{SEM}(\mathrm{n}=3-5) ;{ }^{*} \mathrm{p}<0.05 ;{ }^{* * *} \mathrm{p}<0.001$. 
A

B

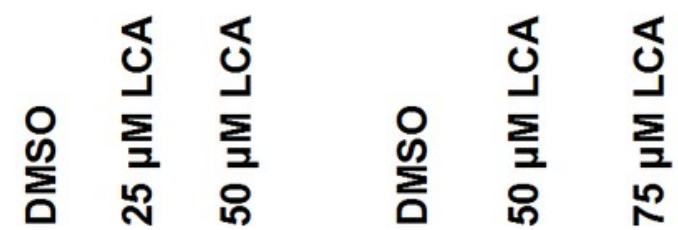
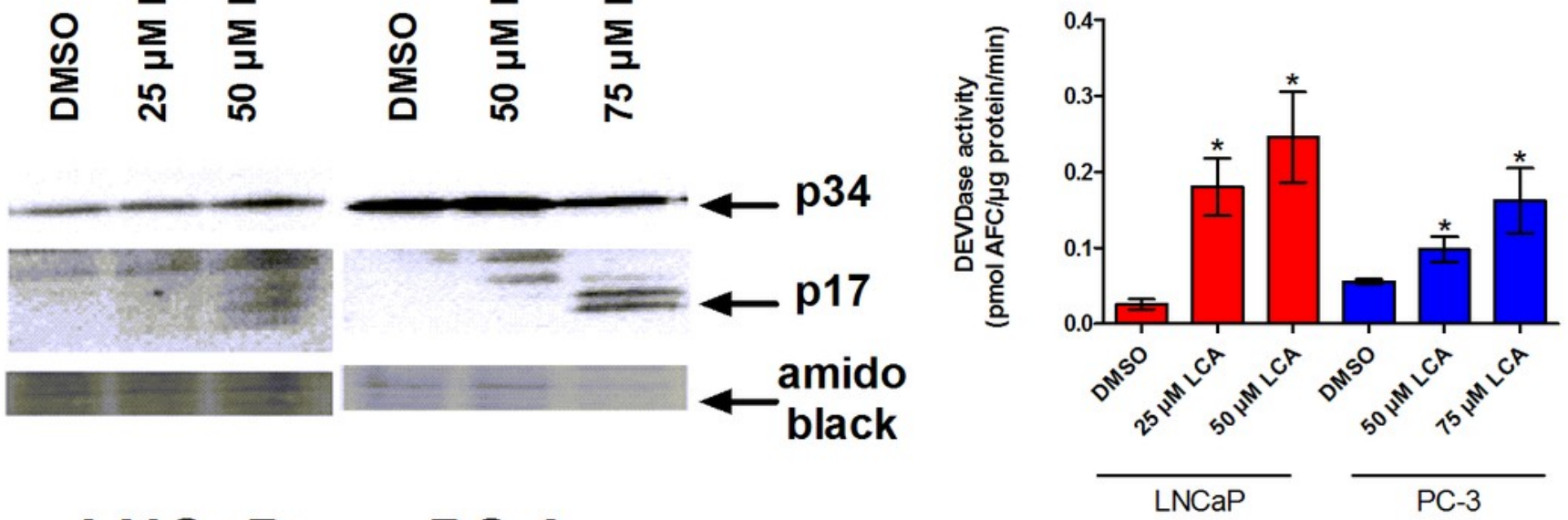

LNCaP PC-3

C

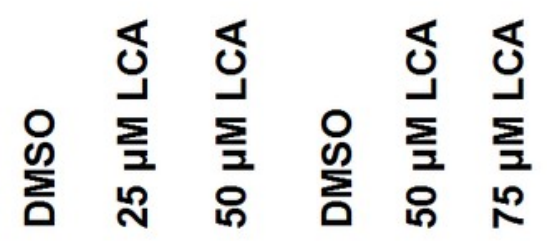

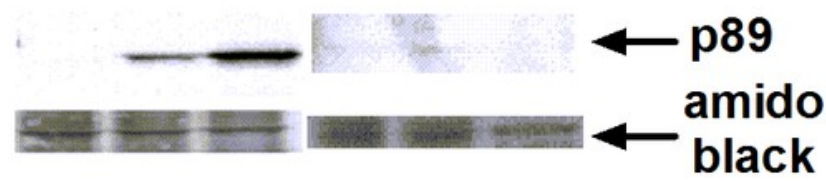

D

LNCaP PC-3

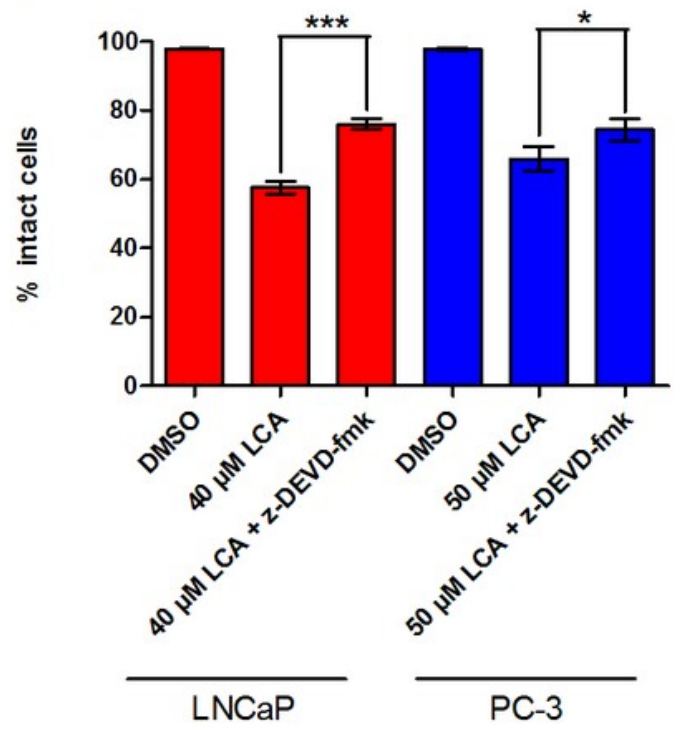




\section{Figure 3}

LCA activates the extrinsic pathway of apoptosis in androgen-dependent and -independent prostate cancer cells.

(A) Activity of caspase-8 was measured by cleavage of the fluorogenic substrate Ac-IETD-AFC after 24 hours of treatment of LNCaP and PC-3 cells with increasing concentrations (25-75 $\mu \mathrm{M})$ of LCA . (B) Inhibition of cell death after a 24 hour co-exposure of LNCaP (40 $\mu \mathrm{M})$ or PC-3 (50 $\mu \mathrm{M})$ cells to LCA and $10 \mu \mathrm{M}$ of the membrane permeable caspase-8 inhibitor z-IETD-fmk. Activities are presented as means $\pm \operatorname{SEM}(\mathrm{n}=3-5) ;{ }^{*} \mathrm{p}<0.05 ;{ }^{* *} \mathrm{p}<0.01$. family: $\gg$

A
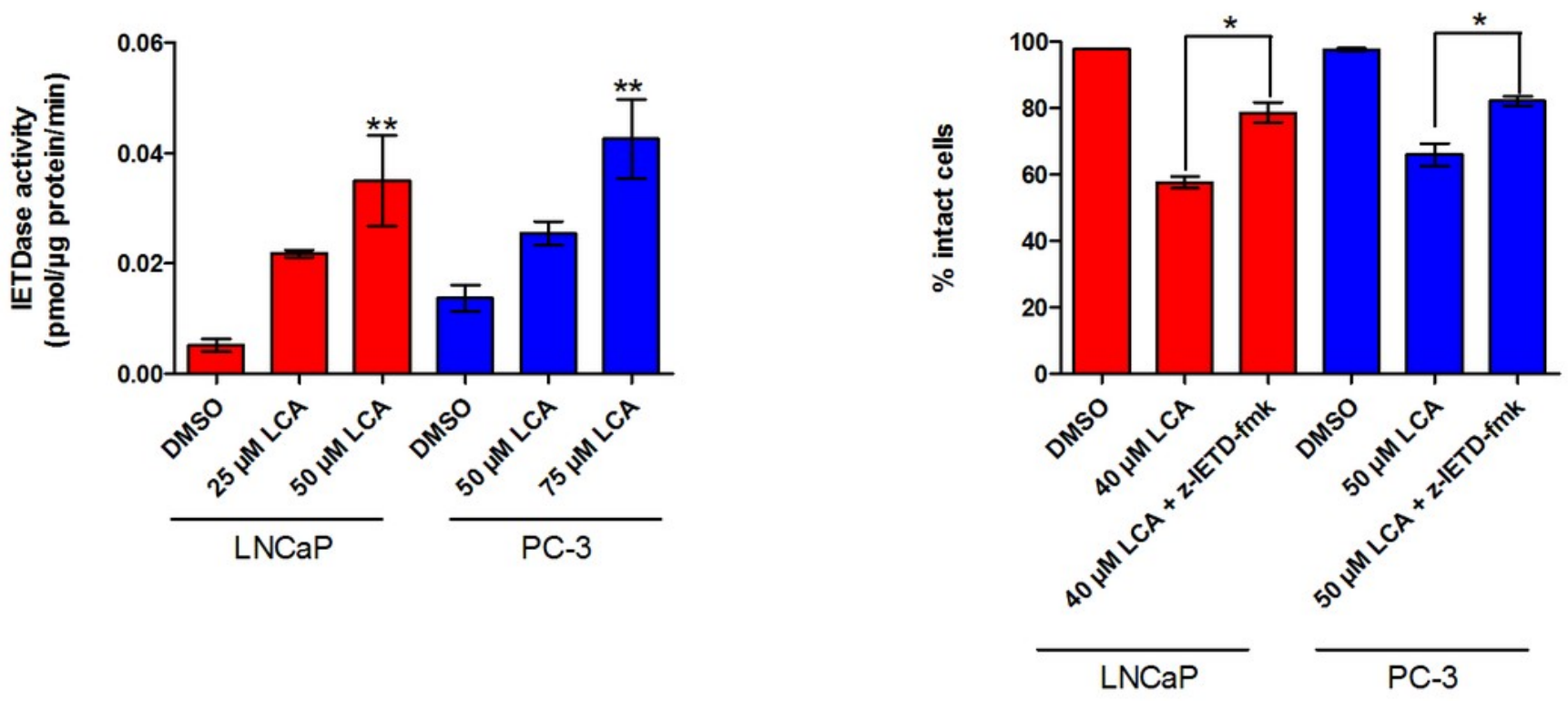


\section{Figure 4}

LCA activates the intrinsic pathway of apoptosis in androgen-dependent and -independent prostate cancer cells.

(A) Activity of caspase-9 was measured by cleavage of the fluorogenic substrate Ac-LEHD-AFC after a 24 hour treatment of LNCaP and PC-3 cells with increasing concentrations (25-75 $\mu \mathrm{M})$ of LCA . (B) Expression levels of Bcl-2 and cleavage of Bax and Bid after a $24 \mathrm{~h}$ exposure of LNCaP and PC-3 cells to increasing concentrations (25-75 $\mu \mathrm{M})$ of LCA . (C) Mitochondrial membrane permeability was measured using TMRE in LNCaP and PC-3 cells treated with 50 and $75 \mu \mathrm{M}$ LCA, respectively. In (A) and $(\mathrm{C})$ responses are presented as means $\pm \operatorname{SEM}(\mathrm{n}=3-5) ;{ }^{*} \mathrm{p}<0.05 ;{ }^{* *} \mathrm{p}<0.01$. 
A

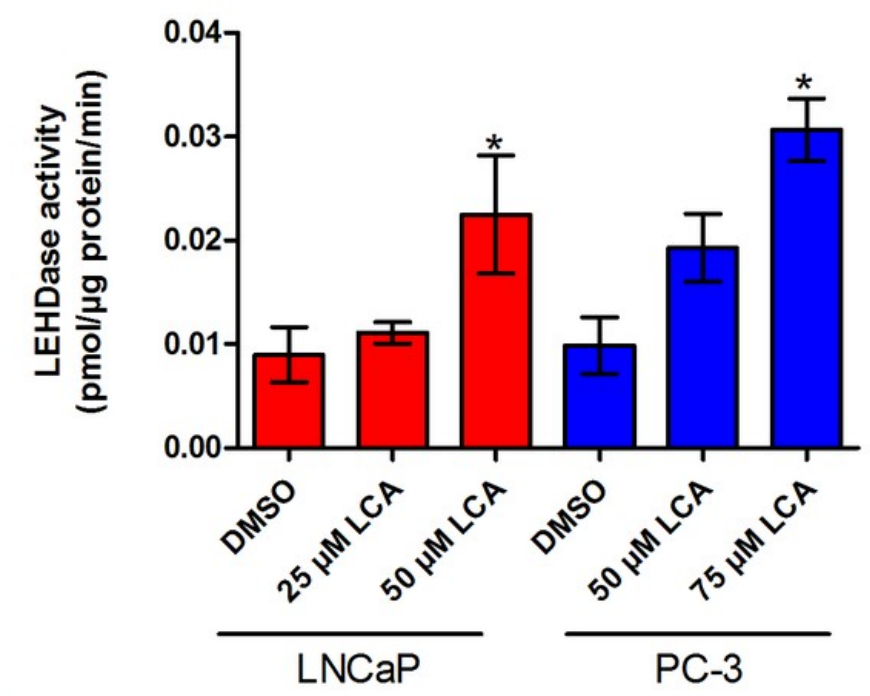

B

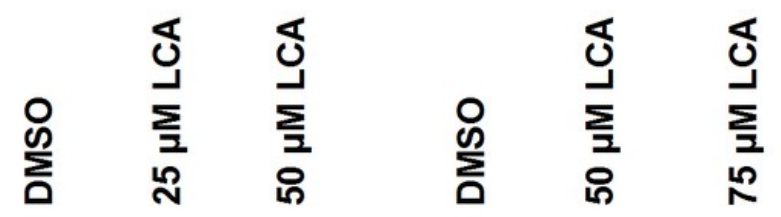

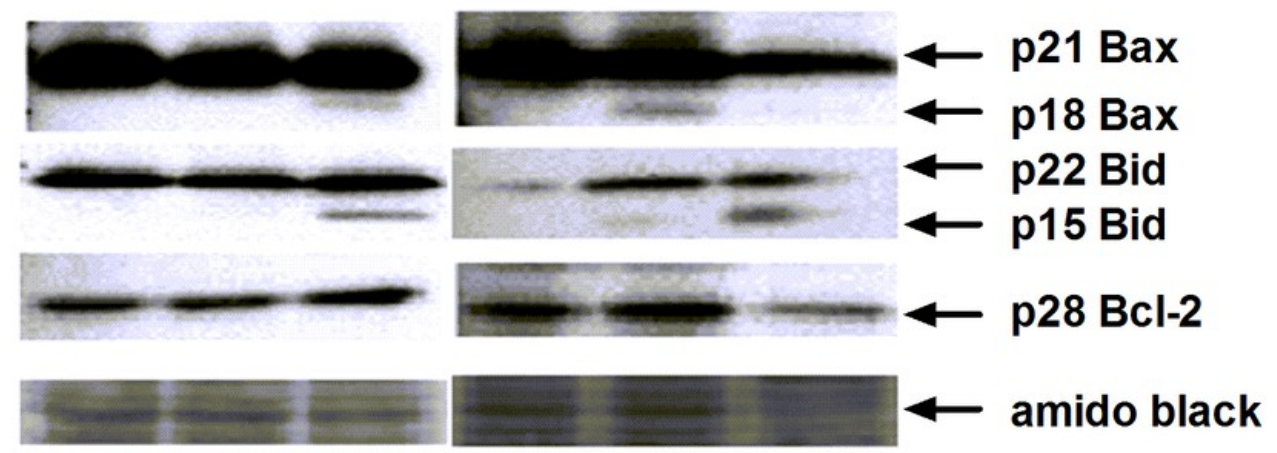

LNCaP

PC-3

C

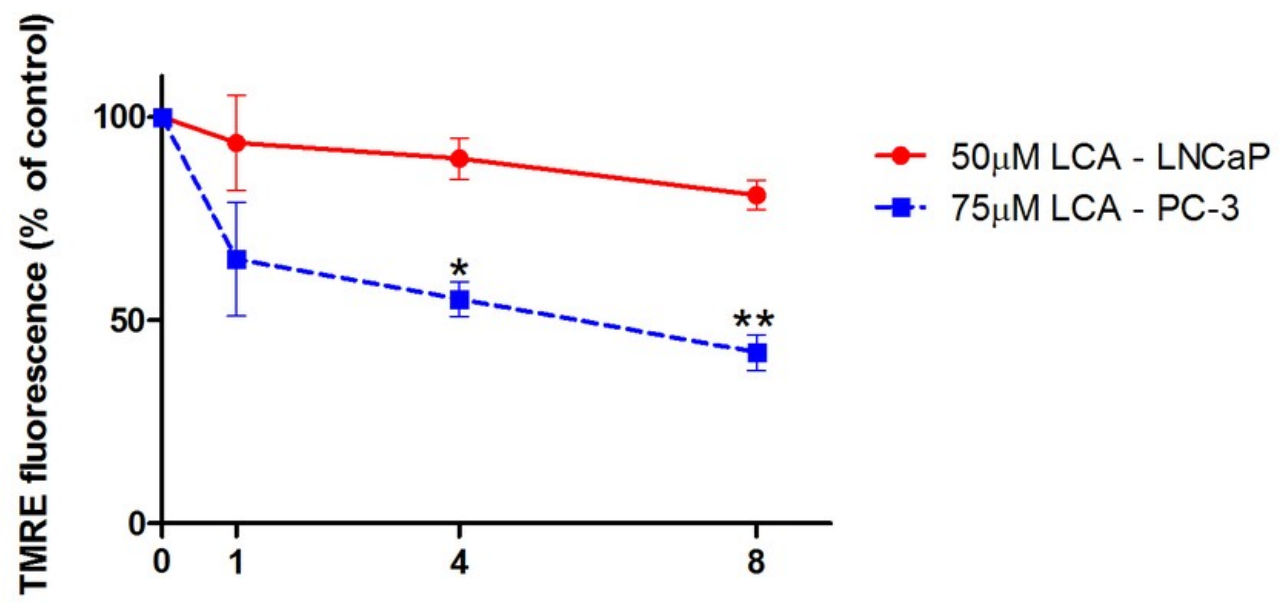

Time (hours) 